\title{
DIE DARSTELLUNG VON EHE UND FAMILIE IN DEN EPISCHEN UND LYRISCHEN DICHTUNGEN DES STATIUS
}

In der "Divina Commedia". im 21. Gesang des purgatorio, schildert Dante, wie er und sein Jenseitsfthrer Vergil Zeuge einer bedeutungsvollen Begebenhelt werden: Bin Beben des Lauterungsberges zeigt an, daB eine dort seit Jahrhunuerten buBende Seele soeben entsuhnt worden 1st; sie kann nun in das Parades eingehen. Diese Seele stellt sich ihnen namentlich vor: Es ist der Dichter statius, der ihnen auch die T1tel selner belden Epen nennt, die "Thebais" und die unvollendete "Achilleig". Wir erfahren ferner, daB der Dichter Statius zu Lebzeiten durch das Vorbild Vergils zur Poesie gelenkt und durch die 4. Ekloge zum wahren Glauben bekehrt worden 1st: "Per te poeta ful. per te cristianon'. Freilich hat or sein Christentum nicht offentich bekannt und dafur lange Jahre im purgatorio gebuBt.

Man hat sich in der modernen Forschung mit Recht die Frage geste11t: Was veranlabte Dante, Statius zum Krypto-Christen zu machen? Zur Antwort wurden vor allem 2 Passagen aus der "Phebals" herangezogen. Die eine ist die Beschreibung des Altars der clementia ${ }^{2}$. Sie brachte man mit dem berthmten Altar auf dem Areopag in Verbindung, der dem unbekannten Gott, dem Agnostos theos, gewe1ht war. Allerdings ist der Vers des Statius "ignotae tantum felicibus araen in seiner Aussage ganz und gar fern von einer Widmung ignoto deo.

Die andere Stelle Pindet sich im 4. Buch der "Thebais" 4 . Poliziano meinte, daB die hier genannte hochste Gotheit der dre1-

1 La divina commedia. Purgatorio XXII 73.

2 Thebais XII 481-518.

3 Thebais XII 496.

4 Thebais IV 514-517. 
Fltigen Welt, "triplicis mundi summum", die Christliche Trinitat bezeichnen solle - ouch dieses oine recht gewaltsame Gleichung ${ }^{5}$.

Schlieblich gab es noch eine weitere Vermutung: Statius pelegte das Grab Vergils bei Neapel zu besuchen. Das Mittelalter kannte jene Tradition, derzufolge auch der Hl. Paulus dieses Grab besucht haben sollte. So mochten die beiden einander begegnet, der heidnische Dichter vom Volkerapostel bekehrt worden sein. Freilich Pindet sich das Detall vom Besuch des Statius am Grabe Vergils nur "Silvae" ${ }^{6}$; de "Silvae" aber waren dem Mittelalter unbekannt, sie wurden erst in der Renaissance im 15. Jh. wiederentdeckt. Alle drei Hypothesen bleiben somit duBerst unmahrscheinlich.

Nun ist andererseits der Platz, den Dante dem Statius in der "Divina Commedia" einrdumt, Pflnffach bedeutsam: 1/ Es 1st der Moment selner Erlosung, in dem Dante und Vergil dem Statius begegnen, der wichtigste Augenblick in der Existenz elnes christilchen Individuums, ein Augenblick, den Dante einzig und allein bei Statius beschreibt. 2/ Im 4. Canto des Inferno erfahren wir, daB Im limbo, dem Rande der Unterwelt, die grobten Dichter sich aurhalten. Es sind 5: Homer, Verg1l, Horaz, Ovid, Lukan. Ihnen wird ausdrucklich Dante als 6. beigestel1t ${ }^{7}$. Jetzt tritt als Letzter Statius hinzu - durch ihn wird die bedeutsame Siebenzahl erftlit, wird die Heptade der høchsten Poeten abgerundet. 3/Vergil, der Dante durch H\&lle und Fegefeuer fahrte, muB kurz vor dem Himmel zurtackbleiben. Seinen platz als Dantes Funrer nimmt Statius ein, der erst gegen Ende des gewaltigen Gedichts durch Beatrice urid hernach durch St. Bernhard abgelost wird. Statius steht so zwischen Vergil und Beatrice, er gehbrt in die Trias der 3 cur Dante wichtigsten Wesen der Welt. 4/ In der Figur des Statius hat Dante offenbar

5 Oratio super Fabio Quintiliano et Statii Sylviis, Angeli Politian1 opera quae extitere, Basileae 1553, 493.

6 Silvae IV 4,53.

7 La divina commedia. Inferno IV 88-102. 
ein Abbild seiner eigenen poetischen Person geschaffen: Es ist die Vergilverehrung, die nahezu religiose Hingabe an den Meister aus Mantua, die beide Poeten auf das innigste verbindet. Von beiden gilt das Fort:

\section{"Die Aeneide, die mir Mutter gewesen is $\hat{\imath}$ und Amme in meiner Dichtung, auch nicht das Kleinste schrieb ich ohne ihr Vorbild"8.}

5/ SchlieBlich ist beobachtet worden, daB der Titel der "Divina Commedial offenbar inspiriert ist durch den Ehrentitel, den Statius am Ende der "Thebais" in der berthmten Sphragis dem Werke Vergils zuspricht: Er nennt es "divinam Aeneidan".

Bei solcher Nahe Dantes zu Statius bleibt allerdings immer noch die Frage offen nach dem Christentum, das der mittelalterliche Dichter dem antiken zuspricht. Wie gezeigt, ist die auBere Evidenz kaum stichhaltig. Es mag so an der Zeit sein, einmal nach der Inneren Evidenz zu fragen: Sind die Opera des Statius derart, daB in Dantes Augen ein Krypto-Christ ihr Autor sein konnte? Hier dare man herk $8 \mathrm{mmlichen}$ Urteilen nicht trauen: Im 19. und Prthen 20. Jh. wurden die Werke des Statius von der Philologie abgewertet als allzu blutrunstig, als grausig und grausam, als dem GraBlichen zugewandt. Tiefer hat da P. Thomas geblickt, der schon 1904 Statius so charakterislérte: "Le plus beau côté du caractère de Stace, c'est la profondeur et la sincérité de seg sentiments de famillo. La piété Plliale, l'amour conjugal, la tendresse paternelle, lul ont insplré des accents émus. Il était né pour être le poéte de la vie domestique" ${ }^{10}$. Hat Thomas mit dieser Charakterisierung recht? Er hat sie selbst nur entworfen, nicht naher begrandet. Lassen Sie uns hier und heute kurz den Komplex zu klaren versuchen.

$$
\mathbf{x} \quad \mathbf{x}
$$

8 La divina commedia. Purgatorio XXI 97-99: "dell'Eneida dico, la qual mamma / fummi e fummi nutrice poetando/ sanzessa non rermal peso di dramma".

9 Thebais XII 816.

10 P.Thomas, Discours rectoral sur le poète Stace, Gent 1904. 
Beginnen wir bei den "S1lvae": 32 Gelegenheitsgedichte. Jeder der 5 Bucher enthalt mindestens e 1 n Beispiel fur unser Thema, Jewells an prominenter Stelle placiert. Gleich nach dem Einleitungsgedicht, das nattrilch den Kalser lelert, folgt in 1,2 eln umfangreiches Epithalamion. Die Bucher 2 und 3 werden abgeschlossen jeweils mit einem Gedicht an die Gattin eines Dichters: 2,7 sucht die Witwe Lukans zu trosten, 3,5 spricht zur eigenen Gattin. Dieses 1etztcenannte Poem erscheint besondera wichtig: Es schlieBt den Zyklus der ersten 3 Bucher, die geschlossen publiziert wurden, und dient so als persinllche Sphragis, als Selbstaussage des Autors, die er jedoch nicht der elgenen person vorbehalt, sondern der Gattin widmet eine exceptionelle Ehrung der Ehorraiu

Im 4. Buch 1st als vorletztes ein Gratulationslied eingeruckt anlablich der Geburt des 3. Kindes des Freundes Menecrates. Statius puhrt es in der einleitenden Prosapraelatio ein als Huldigung rur den Freund, "well or durch die Zahl seiner KInder unserer Heimatstadt Neapel Ehre gemacht hat"11. Das 5. Buch schlieBlich, das orfenbar aus dem NachlaB stame, enthalt/unter insgesamt $5 /$ gleich $3 \mathrm{Ge-}$ dichte, die zum Thema Familie sprechen: 5, 1 die Klage uber den Tod der Frau des Froundes Abascantius, zu der es in der Prosapraefatio ho1Bt: "uxorem vivam amare voluptas est, depunctam religio"12. ES Polgt in 5,3 das lange Trauergedicht auf den Vater, in 5,5, eln ebensolches auf den Tod eines dem Dichter an Kindes statt nahestehonden Knaben. Auch 2,1 ist ein Klagelied fur das geliebte Kind eines Freundes.

So ist in der "Silvae" past ein Viertel der Gedichte - zahlr man die Zeilen, weit mehr als ein Viextel der Vexse - Themen gewidmet, die Parillare Bindungen zum Inhalt haben, Bezlge zwischen Ehegatten, zwischen Sohn und Vater, zwischen pplegeeltern und Kind. Bezelchnend, daB das, was fur die Gelegenheitsgedichte gilt, auch in den Epen aupeallt.

$\mathrm{x} \quad \mathrm{x} \quad \mathrm{x}$

11 Silvae IV 8: Gratulatio ad Jullum Menecratem; cfr Prepatio in librum IV: "cui gratulor quod Neapolim nostram numero liberorum honestaverit".

12 Silvae, prefatio in librum $V$. 
Gerade in dieser Gattung wirde man derartiges kaum vermuten. Und doch beglnnt die unvoliendete "Achlllels", die das ganze Leben des groben Kriegers schildern will, aber nach 1127 Versen abbricht, nicht mit einem martialischen, sondern einem mutterlichen Motiv: Thetis sorgt sich um ihren jungen Sohn, sie sucht ihn mit aller Macht, schlieBlich mit List vom Kampe un Troja Pernzuhalten. Zu diesem Zwecke entPuhrt sie ihn aus seines Lehrers Chiron Hut nach Skyros und versteckt inn dort bei der Fainilie des Inselkonigs, vorkleidet als Madchen, unter den JungPrauen ${ }^{13}$. Es ist gewiB kennzeichnend fur die Mentalitat unseres Dichters, daB er sein Heldenepos aus der Perspektive der mutterlichen Sorge und Fursorge seinen Ausgang nehmen laBt; es pindet seinen Fortgang in dor zqischen Achill und der Konigstochter aufkeimenden Liebe, bis dann der Klang der Kriegstrompete das zarte Idyll abrupt beendet.

Ca. $10 \mathrm{mal}$ so lang wie die unvollendete "Achilleis" ist das andere Epos des Statius, die 12 Bthcher umfassende "Thebais". Dieses 'Lied von Theben' 1st, sieht man genauer $z u$, vom Dichter konz1plert als Schilderung nicht des Zuges der "Sieben gegen Theben" oder der Schicksale einzelner Protagonisten aus der Stadt des Kadmos: Thema ist vielmehr "das zerrattete Haus, die fluchbeladene Famllie des Ödipus"14. Dementsprechend geht die Darstellung auch eber den 2 welkampe der Odipussøhne Eteokles und Polyneikos hinaus und beschreibt In welteren 1000 Versen auch noch das Schicksal Antigones und Rreons. Erst dann findet die groBe Familiensaga ihr Ende.

Neben dem Hauptthema weisen auch nicht wenige Details und Episoden in den Bereich der Familienthematik. So berthrt die Erzahlung vom Gattenmord aup Lemnos ein zutiefst traglsches Ehethema ${ }^{15}$, endet aber doch durch die Verbindung zur Argonautensage und mit ihnen in zuversichtlichem Tone. Auch die Fabel von Hypsipyle selbst hat ihr Zentrum in der Trennung der Mutter von ihren Kindérn und kulminiert

13 Achilleis I 25 Pf.

14 Thebais I 16-17:"... limes mihi carminis estol oedipodae confusa domus".

15 Thebais V 17-498. 
Im Glatck der Wiedervereinigung ${ }^{16}$.

In der Erzahlung selbst fahrt die Schilderung der Doppelhochze1t des Polyneikes und des Tydeus zu einem ersten glanzvollen Høhepunk ${ }^{17}$. Buch 3. endet $/ 678 / \mathrm{mit}$ der rahrenden Szene, in der des Polyneikes Weib ihren Vater um Hilfe fur den Gatten anfleht. Im Katalog der Streiter, einem alten epischen topos, hat Statius als besonderen Akzent an den AbschluB die Begegnung gesetzt ${ }^{18}$, die geradezu an eine christliche Kreuzwegstation erinnert: Der junge Parthenopaus begegnet seiner welnenden Mutter, die thn vom Gang in den Krieg und in den Tod abhalten møchte und es doch nicht vermag.

Âhnlich ist es auch in den Kamplen, die in der 2. Halfte des Epos geschildert werden: oft und oft werden familiare Beziehungen betont, werden Szenen aus diesem Bereich eingeftgt. Mag auch dem Statius ein Bild wie Hektors Abschied von Andromache bei Homer nicht gelungen sein, sein Epos enthalt doch genung anrahrende Begegnungen und Gesprache, in denen das Grauen des Kymplens und Sterbens der ramiliaren Geborgenheit eindringlich gegentbergestellt wird. Ergreifend die Todesszene des Crenaeus, endend mit den Zeilen:

"Als letzter Ruf erklang vom ersterbenden Munde das Wort "Mutterl", und sghon erstickten die Fluten den Aufschrei des Ármen"19.

Ähnlich anruhrend ist die Todesszene des Parthenopaeus, der seiner fernen Mutter gedenkt und dem Freund auftragt, sie zu trosten 20 - nicht umsonst wird am Ende des Epos gerade an ihn mit dreffacher Anapher erinnert "Arcada"21, an ihn, den Freund und Feind gleichermaBen beweinen, dessen von seiner Mutter so sehr geliebte, nun unnatz geopferte Jugendliche Schønheit noch im Tode erhalten 1st,

16 Thebais V 710 Pf.

17 Thebais II $201 \mathrm{fP}$.

18 Thebais IV $309 \mathrm{ff}$.

19 Thebals IX 349-350: "Ultimus ille sonus moribundo emersit ab ore:/ "materl" in hanc miseri ceciderunt flumina vocem".

20 Thebais IX $885 \mathrm{PP}$.

21 Theba1s XII 805-807: "Arcada quo planctu genetrix Erymanthia clamet, I Arcada, consumpto servanten sanguine vultus, I Arcada, quem geminae pariter flevere cohortes". 
der so als letztes Bild des groBen Gesanges von Theben dem Leser vor Augen bletbt.

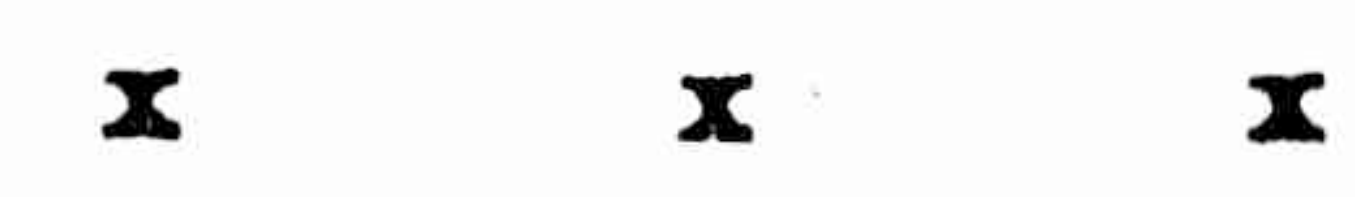

Auch wenn wir langst nicht alle Angaben anfthren konnten, so mag doch vielleicht andeutungsweise deutlich geworden sein, welch eine wichtige Rolle das Thema der Familie im gesamten Werke des Statius spielt. Daran knapft sich nun die Frage nach seiner eigenen Einstellung zu diesem ihm selbst so wichtigen Themenbereich an. Wir konnen hier nur kurz einiges herausheben, da entsprechende Vorarbeiten nich vorliegen. Über seine eigene the sagt Statius:

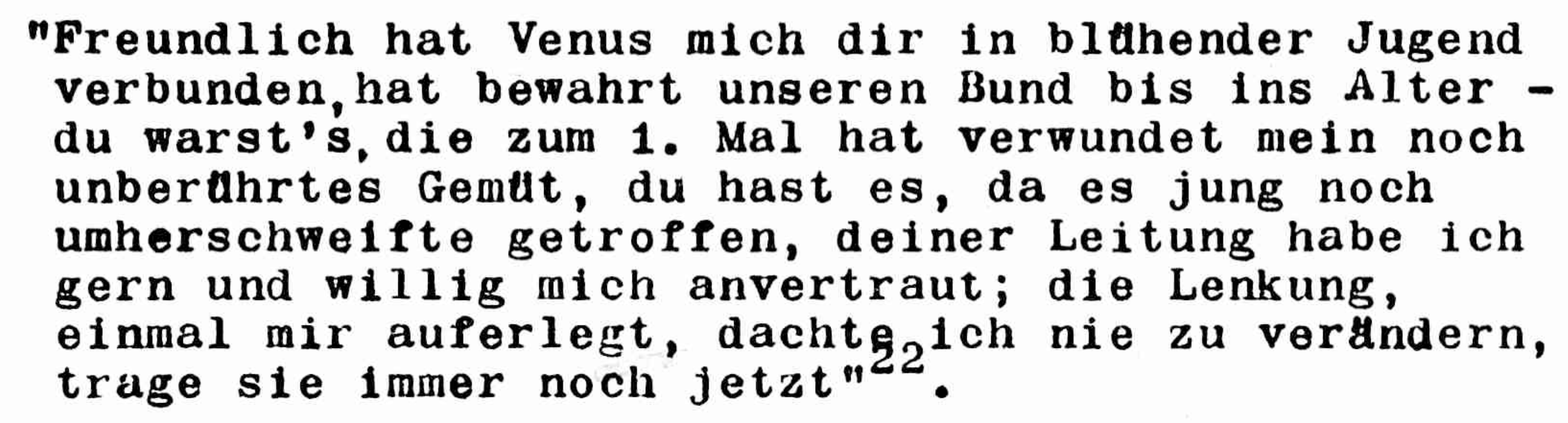

Man wird in diesen liebevollen Worten das Bild einer idealen Ehe sehen durfen, vielleicht das einer 1dealisierten, poetisch verklarten - doch jedenfalls auch das eines bestandigen Bundes, dessen gefestigte Substanz im folgenden mit vielen E1nzelheiten belegt wird ${ }^{23}$. Diesem hohen Anspruch Fird auch der neugeschlossene Ehebund des Freundes Stella angenthert, besungen in dea Epithalamion ${ }^{24}$. Stark betont wird die Legalitat des Bundes:

22 Silvae III 5,22-28: "... etenim tua - nempe benigna"/quam mihi sorte Venus iunctam florentibus annis/servat et in senium - tua, quae me vulnere primolintactum thalamis et adhuc iuvenile vagantem fixisti, tua frena libens docilisque recepi, let semel

insertas non mutaturus habenas/usque premo ..."22

23 Ibidem, 27-42.

24 Silvae I 2: Epithalamion in Stellam et Violentillain. 
Gesetze regeln diese Liebe, sie ist nicht ungezllgelt/leges et Irena/, sie ist dem Brauch und der Scham unterworien/ianitor aut lex aut pudor/25. In dem an Lukans Witwe Polla gerichteten Lied 1st der Katalog ihrer Vorzlige bemerkenswert. Sie zoichnet sich aus durch:

"Schornheit, Einfachheit, Freundlichkeit, Besitz, Charm, Adel, Lieblichkeit"26

sie besitzt zugleich auch eine Blidung glelch der ibres Gacten. Der SchluB des Gedichtes betont, daB die Witwe den toten Gatten nicht in nichtigen magischen Brachen verehrt, sondern ihn selbst, sein personliches Wesen weiterhin an ihrer Seite weiB:

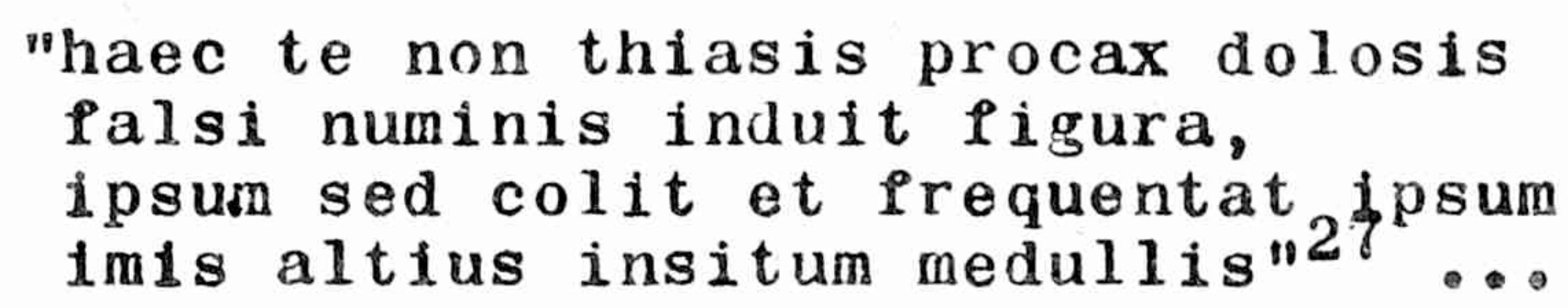

Sieht man von der obligatorischen offiziellen Huldigung an den Machtigen des Tages, an den Kalser Domitian, in $I$, 1 ab, so rahmen die 3 Ehebilder das Corpus der 3 ersten Silvenbucher: I, 2 besingt die beginnende the eines Freundes, II, 7 die vergangene des Dichterfreundes Lukan, III, 5 die eigene Ehe des Dichters Statius im Moment ihrer Bewlhrung.

Schauen $1 \mathrm{r}$ uber diese Trias hinaus, so sehen wir in IV, 7 die Geburt des 3. Kindes oines Freundes als AnlaB, die Freuden Pamiliarer Bindungen zu Peiern. Diesem Freudenlied stehen die Trauergestinge aus Buch 5 entgegen ${ }^{28}$. Zu ihnen geselit sich noch II, 1 , ebenfalls an exponierter stelle, ein Trauerlied, geschapfen pur den toten pelegesohn eines Freundes. In allen 4 Gesangen wird immer wieder die Enge der Pamiliaren Bande zwischen dem Verlorenen und

25 Ibidem 24-37.

26 Silvae II 7,83-85: "Forma, simplicitate, comitatelcensu, sanguine, gratia, decore ... doctam atque ingenio tuo decoram".

27 Ibidem, 124-127.

$28 \mathrm{Nr} 1,3,5$. 
den Verlassenen betont, wird die Tiefo der gegenseitigen Liebe und d10 Schwere des Verlustes nachdrelcklich herausgestellt. Symptomatisch der Satz:

\begin{abstract}
"Wahrlich kein Wunder, daB Brust euch an Brust hat vorbundon innig vereint lange de EINTRACHT in ununterbrochener Folge" 29 .
\end{abstract}

Wichtiger noch, daB Statius die Einehe als hohes Gut preist:
"Stammiest du auch aus bestem Haus, wargt schyn, we ein Gatte es nur wanschen kann, - groBer noch ist deine Ehre: ein einzlges Ehebett kanntest du nur, nur eine einzige liebe nahrtest verborgen du in deinem Herzen"30.

Elfer und liebevolle Zuneigung der Gattin werden gerthmt:
"Selbst tragt sie Sorge fur den Bedare lhres Gemahls, mahnt ihn zum Werk und macht es ihm leichter 31 .

Nicht nur die Gattenliebe indet in Statius lhren inspirierten - und inspirierenden - Sanger, auch die Beziehungen zwischen Eltein und Kindern werden von ihm poetisch verkltrt. In den "Silven"geschioht das in den Trauerliedern auf den eigenen Vater 32 und auf die Prlegekinder ${ }^{33}$. Das Gedicht auf den Vater $18 t$ mit fast 300 Versen /genau: 293/ das lingste der Trauerlieder in den "Silven". Es muB daruber hinaus auch dem Dichter selbst besonders am Herzen gelegen haben, ersoleint es doch erst $1 \mathrm{~m}$ V. Buch der "Silven", offenbar aus

29 Silvae $V$ 1,43-44: "Nec mirum, si vos collato pectore mixtos/ lundit inabrupta Concordia longa catena".

30 Ibidem, 53-56: "... tibl quamquam et origo niteretlet pelix species multumque optanda maritis, / ex te maior honos unum novisse cubile,|unum secretis agitare sub ossibus ignem".

31 Ibidem, 119-120: "... Povet anxia curas lconiugis hortaturque simul flectlque labores".

32 Silvae V 3: Epicedion in patrem suum.

33 Silvae II 1: Glaucias Atedii Melioris dedicatus, und V 5: Epicedion in puerum suum. 
dem NachlaB herausgegeben, volle 15 Jahre nach dem Tode des Beklagten. Da es einerseits angibt, 3 Monate nach dem Ableben des Vaters geschrieben $z u \operatorname{sein}^{34}$, andererseits spatere Ereignisse erwahnt, hat Statius es offenbar auch einmal oder méhrmals umgearbeitet.

Neben diesen auBeren Zeichen der auBergew nahme des Autors stehen auch zahlreiche Aussagen des Textes, die seine innige Beziehung zum Vater bezeugen, der thm auch Vorbild und Lehrer in der Poesie gewesen war ${ }^{35}$. Noch ein Detail von Bedeutung: Der Sohn ruhmt, daB der Vater nur eine elnzige Ehe eingegangen 1st:

"Der Ehebund ward dir bekannt in einer einzigen Fackel, eine einzige Liebe hat dich beseelt"36.

Solche Hochschatzung der Ehe und der Faisilie seitens des Statius sind jedoch nicht isoliert zu sehen. Sie flgen sich vielmehr auch oin in die Bestrebungen seiner Zeit. Es ist bekannt, daB gerade whrend der Lebenszeit des Statius, unter der Regierung des Domitian, starke Bestrebungen zur Wahrung und zum Schutze der Familie im Gange waren, daB man sich um die Hebung der befentlichen Moral, um die Besserung der Sitten bemtihte.

An Einzelbeispielen sei orwhnt, daB man nach langer Zeit wieder Prozesse gegen schuldige Vestalinnen durcheahrte, daB Domitian selbst die Kastration von Knaben ausdrticklich verbot und Ehegesetze erlassen wurden. Es sei auch an das lus trium liberorum erinnert, durch das Kinderrelchtum steuerlich gefordert wurde, oder an die Bacher Martials, die sich ihrer Anstandigkeit rahmen. So steht Statius nich vereinzelt da, sondern pügt sich ein in eine leihe von Haltungen und Handlungen, die seinen eigenen Intentionen und ÄuBerungen parallel laufen. Freilich ist er derjenige, der diesen Gedanken die poetisch schönste, gedanklich beeindruckendste Form zu verlelhen vermochte - eine Form, die selbst noch nach so vielen Jahrhunderten den modernen Leser anzusprechen und anzuregen vermag.

$\mathbf{x} \quad \mathbf{x}$

34 Silvae V 3,29-30.

35 Ibidem, $209 \mathrm{ff}$.

36 Ibidem, 240-241: "... una tibi cognita taeda/conubia, unus amor". 
Um unsere Betrachtungen nicht aber Gebahr auszudehnen, verzichten wir hier auf Beispiele aus den beiden Epen des Statius, die zahlreich genug die namlichen Gedanken bekraftigen konnten. Es mag vielmehr an der Zeit sein, zum Ausgangspunkt unserer Überlegungen zurlickzukehren. Was kann, so haben wir gefragt, Dante, den aufmerksamen Leser und einfuhlsamen Nachfolger des Statius, bewogen haben, diesen als Krypto-Christen zu charakterisieren? Gewi er mag eine Legende vorgefunden haben, die ihm derartiges suggeriorte. Aber die Frage blelbt, ob thm vielleicht auch Elemente der Werke des Statius den Glauben an seine Christianitat nahelegten. Sicherheit wird sich in dieser Frage kaum gewinnen lassen. Aber oine Vermucung zu formulieren, møge verstattet sein.

Wie die zuvor aufgefuhrten Testimonien zeigen, hat Statius sich bemorkenswert oft dem Thema der Familie zugowandt. Er hat dabei einen Standpunkt zum Ausdruck gebracht, der sich wohl aus heidnischer Ethik herleitete, der dem Christen Dante aber aus seiner Sicht in vielfaltiger Hinsicht vertraut klingen muste. Lobpreis der Jung frallichkeit vor der the und der Bestandigke1t in der Ehe sind dem Statius sicher nicht durch die Hl. Schrift nahegebracht morden - doch der Christ Danto wird sie vermutich in seiner Foise als christliches Gedankengut angesehen haben. Um nicht mibverstanden zu werden: Es soll hier weder die These vertreten werden, daB Statius wirklich ein geheimer Christ war, noch auch, daB Dante thn allein aufgrund seiner stellung zu den Fragen und Formen von the und Familie als solchen gesehen hat. Auch wollen wir nicht postulieren, daB Dante neben den von thm genannten Epon des Statius auch desson S1lven gekannt hat. All das liegt uns fern. Aber dio Hypothese hat doch olnen gewissen Grad von plausibilitat par sich: Statius, dor "poéte de la vie domestique", machte es gerade durch diseo seino Haltung dem Mittelalier und speziell dem Dichtor Dante leicht, Ihn als Kenner und viellelcht sogar als Anhanger der christichen Hellslehre und speziell ihror Familienethik zu sehen.

Mir scheint, is verhalt sich hior analich wo bel den ingierten Brwefwechsel. zwischen dem Apostel Paulus und den Phllosophen Seneca: In bolden fallen wird von dor spateren Tradtiton elne noble pagano igur des alten kom in die gelotige Nithe dos aupachsenden Christentums gerluckt. Wir sehen heute, daB vorwande Thesen zu 
moralischen Fragen doch auf der einen Seite aus der offenbarung, auf der anderen aus der Vernunftreligion der stoa begrandet wurden und daher bel AuBerlicher Ähnlichkeit innerlich sich fundamental unterscheiden.

Aber daB das Konstrukt einer solchen Kommunikation uberhaupt moglich war, zeigt, wie die Lehren des Christentums im mancher Hinsicht den besten Geistern des Heidentums in jener Zeit aus dem Herzen sprachen. Gerade an der Frage der Familie erweist sich, wie die 'Palle der Zeit' die Menschen bewegte und zueinander fahrte - der "Christ" Statius ist sicher nur eine Fiktion, historisch gesehen; doch geistesgeschichtlich ist or eine bedeutsame und stimmige Symbolfigur.

Bernhard Kytzler Freie Universitat Berlin 